\title{
A short note on methods of ranking fuzzy numbers in risk analysis problems
}

\author{
Alireza Sotoudeh-Anvari ${ }^{a^{*}}$ \\ ${ }^{a}$ Department of System and Industrial Engineering, Industrial Management Institute, Tehran, Iran

CH R O N I C L E A B S T RA C T \\ Article history: \\ Received: November 302018 \\ Received in revised format: De- \\ cember 282018 \\ Accepted: March 62019 \\ Available online: \\ March 62019 \\ Keywords: \\ Risk assessment \\ Ranking fuzzy numbers \\ Risk analysis
}

\section{Introduction}

Project management has become a key business process on strategic as well as operational level. Although nearly all projects have restrictions in time, cost, scope and quality, there is a high level of uncertainty in any project too (Perminova et al., 2008). On the other words, risk is inherent in each project and cannot be fully eliminated (Nieto-Morote \& Ruz-Vila, 2011). According to PMBOK, project risk is: "an uncertain event or condition that has a potential effect on at least one project objective" (Perminova et al., 2008). The application of fuzzy logic, introduced by Zadeh (1965), to risk analysis seems very proper; because such analysis is very subjective and connected to vague information (Ngai \& Wat, 2005). Mathematically, fuzzy sets support linguistic vagueness effectively and also provide approximate but very useful descriptions for ill-defined problems (Motawa et al., 2006). Numerous studies have indicated that fuzzy tools are becoming very popular in risk analysis problems because of the flexibility and efficiency they give to a decision maker (e.g. Chen and Chen, 2008; Akyar et al., 2013; Madhuri et al., 2014 and Alidoosti et al., 2012).

Literature shows that fuzzy numbers have been employed usefully to describe the fuzziness in risk analysis context. However, ranking and comparing of fuzzy number is a big challenge. Mathematically, fuzzy numbers are defined by possibility distribution and can overlap each other. As a result,

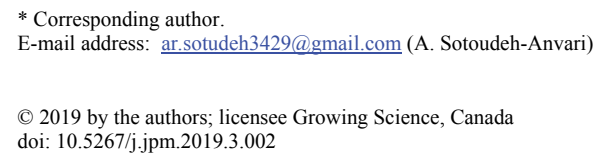


it is not easy to specify which one is bigger or smaller (Kumar et al., 2011). Although several methods have been suggested for ranking fuzzy numbers, nearly all of them suffer from important shortcomings (Sotoudeh-Anvari et al., 2017). On the other hand, some papers have suggested alternative approaches namely, turning fuzzy number into real number by defuzzification. However, according to Zhü (2014), the aforementioned approaches lack validity. It is important to mention that on the basis of an incorrect ranking method, the best choice may be replaced with the worst alternative in a decision problem. Therefore, ranking and comparing fuzzy number is one of the most important tasks in the risk analysis problems under uncertainty. In this short note, we review briefly the methods for ranking fuzzy number in risk analysis.

\section{Research methodology}

We searched SCOPUS and Google Scholar to gather the related papers and to observe the citation of each paper. Then, we searched various keywords such as "ranking fuzzy number", "risk analysis", "fuzzy risk analysis", etc. in these databases. Let us recall that we restricted the time of this short review to 2000-2018. Finally, the 10 most cited papers in this context were selected. We select Chen and Chen (2003), Chen and Chen (2007), Chen and Chen (2009), Wei and Chen (2009a), Chen and Sanguansat (2011), Wei and Chen (2009b), Chen and Wang (2009), Xu et al. (2010), Chen et al. (2012) and Jiang et al. (2015) due to an important influence on the use of ranking fuzzy number in risk analysis indicated by a large number of citations in Google Scholar. Although each method has its own advantages, none is perfect. For example, Kumar et al. (2011) pointed out some drawbacks of Chen and Chen (2009). Also Sotoudeh-Anvari et al. (2017) cleared various weaknesses of Chen and Chen (2009) and Chen and Sanguansat (2011). Recently, Chen et al. (2012) suggested a new ranking technique using areas of positive and negative side and the centroid points of generalized fuzzy numbers and used this method for risk analysis. Although Chen et al. (2012) revealed that Chen and Chen (2007) and Chen and Chen (2009) suffer from some shortcomings, in this note, we indicate that Chen et al.'s (2012) method for ranking fuzzy numbers does not work well. Therefore, the results of risk analysis, provided on the basis of this ranking method, are not reliable.

In the next section, we provide a numerical example to present the problem of Chen et al. (2012).

\section{Shortcoming of Chen et al. (2012) approach}

Suppose that $A_{1}=\left(\mathrm{a}_{1}, b_{1}, c_{1}, d_{1} ; w_{1}\right)$ and $A_{2}=\left(\mathrm{a}_{2}, b_{2}, c_{2}, d_{2} ; w_{2}\right)$ are two trapezoidal fuzzy numbers. Then, we have:

$$
\begin{aligned}
& -\tilde{A}=(-d,-c,-b,-a, w) \\
& A_{1} \oplus A_{2}=\left(a_{1}+a_{2}, b_{1}+b_{2}, c_{1}+c_{2}, d_{1}+d_{2}, \min \left(w_{1}, w_{2}\right)\right) \\
& A_{1} \ominus A_{2}=\left(a_{1}-d_{2}, b_{1}-c_{2}, c_{1}-b_{2}, d_{1}-a_{2}, \min \left(w_{1}, w_{2}\right)\right)
\end{aligned}
$$

Let us recall that although Chen et al. (2012) suggested a ranking method for generalized fuzzy numbers with different left and right heights, they used traditional trapezoidal fuzzy number $\left(\mu_{L}=\mu_{R}=1\right)$ in all numerical examples in that paper. Also ranking of generalized fuzzy numbers by Chen et al.'s (2012) approach relies on the height of fuzzy numbers. However, to validate the efficiency of suggested method only normal trapezoidal fuzzy numbers have been used by the authors (except set 4). Using a classical example, the drawback of Chen et al.'s (2012) approach is pointed out.

Example 1: let $A=(0.1,0.3,0.3,0.5 ; 0.8)$ and $B=(0.1,0.3,0.3,0.5 ; 1)$ be generalized trapezoidal fuzzy numbers (set 4 of Chen et al. (2012), set 4 of Chen and Sanguansat (2011), set 4 of Jiang et 
al. (2015) etc.) (please see Fig. 1). According to Chen et al.'s (2012) approach, we have $B \succ A$. Due to the reasonable properties of fuzzy numbers, suggested by Wang and Kerre (2001), we can logically deduce $B \succ A \Rightarrow B \ominus A \succ A \odot A$. However, by Models (1), (2) and (3), we have:

$B \odot A=(0.1,0.3,0.3,0.5 ; 1)-(0.1,0.3,0.3,0.5 ; 0.8)=(-0.4,0,0,0.4 ; \min (1,0.8)=(-0.4,0,0,0.4 ; 0.8)$

and

$A \ominus A=(0.1,0.3,0.3,0.5 ; 0.8)-(0.1,0.3,0.3,0.5 ; 0.8)=(-0.4,0,0,0.4 ; \min (0.8,0.8)=(-0.4,0,0,0.4 ; 0.8)$.

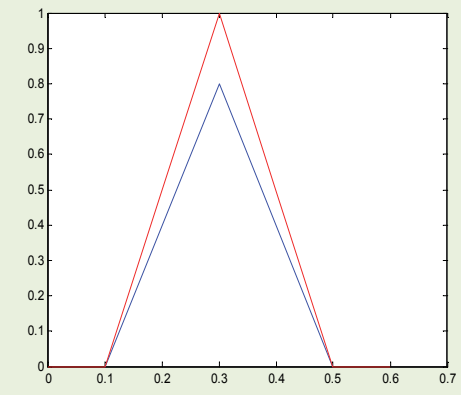

Fig. 1. Fuzzy number in Example 1

On the other words, in this example, $B \succ A \not \nexists B \ominus A \succ A \ominus A$ namely, this result is inconsistent with Wang and Kerre's (2001) reasonable properties. Noteworthy, example 1 has been used in nearly all the aforementioned papers (e.g. Chen \& Sanguansat (2011), Chen and Chen (2007), Chen and Chen (2009), Jiang et al. (2015), etc.). However, the majority of those methods produced a wrong result which is contradiction on the basis of Wang and Kerre (2001). Why does this problem happen? Some researchers such as Liou and Wang (1992), Kumar et al. (2011) and SotoudehAnvari (2016), Sotoudeh-Anvari et al. (2017) mentioned that ranking approaches are independent of the normality of fuzzy numbers. In other words, they pointed out that comparing fuzzy numbers does not depend on the height of fuzzy numbers. It seems true. For example, Kumar et al.'s (2011) ranking technique satisfied all reasonable properties for fuzzy numbers suggested by Wang and Kerre (2001). However, in all of the above ranking methods (e.g. Chen and Chen (2003), Chen and Chen (2007), Chen and Chen (2009), Wei and Chen (2008), etc.), the ranking approach depends on the height of the fuzzy numbers. Hence, the ranking methods proposed by authors are not flawless and the results of risk analysis problems, derived by them, are not valid. However, many papers have applied some of the inefficient ranking methods in the fuzzy risk analysis. Readers are referred to Sotoudeh-Anvari et al. (2017) for more details.

\section{Conclusion}

The literature on the methods for comparing of fuzzy number in risk analysis was briefly reviewed. We can observe that this is an important context that is gaining interest. However, still, more research is needed. We hope that researchers who employ ranking of fuzzy numbers in risk analysis would understand the pitfalls associated with some of these ranking methods.

\section{References}

Akyar, E., Akyar, H., \& Düzce, S. A. (2013). Fuzzy risk analysis based on a geometric ranking method for generalized trapezoidal fuzzy numbers. Journal of Intelligent \& Fuzzy Systems, 25(1), 209-217.

Alidoosti, A., Jamshidi, A., Yakhchali, S., Basiri, M., Azizi, R., \& Yazdani-Chamzini, A. (2012). Fuzzy logic for pipelines risk assessment. Management Science Letters, 2(5), 1707-1716.

Chen, S. J., \& Chen, S. M. (2003). Fuzzy risk analysis based on similarity measures of generalized fuzzy numbers. IEEE Transactions on fuzzy systems, 11(1), 45-56.

Chen, S. J., \& Chen, S. M. (2007). Fuzzy risk analysis based on the ranking of generalized trapezoidal fuzzy numbers. Applied intelligence, 26(1), 1-11.

Chen, S. J., \& Chen, S. M. (2008). Fuzzy risk analysis based on measures of similarity between interval-valued fuzzy numbers. Computers \& Mathematics with Applications, 55(8), 1670-1685. 
Chen, S. M., \& Chen, J. H. (2009). Fuzzy risk analysis based on ranking generalized fuzzy numbers with different heights and different spreads. Expert systems with applications, 36(3), 6833-6842.

Chen, S. M., Munif, A., Chen, G. S., Liu, H. C., \& Kuo, B. C. (2012). Fuzzy risk analysis based on ranking generalized fuzzy numbers with different left heights and right heights. Expert Systems with Applications, 39(7), 6320-6334.

Chen, S. M., \& Sanguansat, K. (2011). Analyzing fuzzy risk based on a new fuzzy ranking method between generalized fuzzy numbers. Expert Systems with Applications, 38(3), 2163-2171.

Chen, S. M., \& Wang, C. H. (2009). Fuzzy risk analysis based on ranking fuzzy numbers using $\alpha-$ cuts, belief features and signal/noise ratios. Expert systems with applications, 36(3), 5576-5581.

Jiang, W., Luo, Y., Qin, X. Y., \& Zhan, J. (2015). An improved method to rank generalized fuzzy numbers with different left heights and right heights. Journal of Intelligent \& Fuzzy Systems, 28(5), 2343-2355.

Kumar, A., Singh, P., Kaur, P., \& Kaur, A. (2011). A new approach for ranking of L-R type generalized fuzzy numbers. Expert Systems with Applications, 38(9), 10906-10910.

Lee, L. W., \& Chen, S. M. (2008). Fuzzy risk analysis based on fuzzy numbers with different shapes and different deviations. Expert Systems with Applications, 34(4), 2763-2771.

Liou, T. S., \& Wang, M. J. J. (1992). Ranking fuzzy numbers with integral value. Fuzzy Sets and Systems, 50(3), 247-255.

Madhuri, K. U., Babu, S. S., \& Shankar, N. R. (2014). Fuzzy risk analysis based on the novel fuzzy ranking with new arithmetic operations of linguistic fuzzy numbers. Journal of Intelligent \& Fuzzy Systems, 26(5), 2391-2401.

Motawa, I. A., Anumba, C. J., \& El-Hamalawi, A. (2006). A fuzzy system for evaluating the risk of change in construction projects. Advances in Engineering Software, 37(9), 583-591.

Ngai, E. W., \& Wat, F. K. T. (2005). Fuzzy decision support system for risk analysis in e-commerce development. Decision Support Systems, 40(2), 235-255.

Nieto-Morote, A., \& Ruz-Vila, F. (2011). A fuzzy approach to construction project risk assessment. International Journal of Project Management, 29(2), 220-231.

Perminova, O., Gustafsson, M., \& Wikström, K. (2008). Defining uncertainty in projects-a new perspective. International Journal of Project Management, 26(1), 73-79.

Sotoudeh-Anvari, A. (2016). Comparing trapezoidal fuzzy numbers by using a hybrid technique on the base of the ideal points and the centroid point. Journal of Intelligent \& Fuzzy Systems, 30(6), 3099-3109.

Sotoudeh-Anvari, A., Sadjadi, S. J., \& Sadi-Nezhad, S. (2017). Theoretical Drawbacks in Fuzzy Ranking Methods and Some Suggestions for a Meaningful Comparison: An Application to Fuzzy Risk Analysis. Cybernetics and Systems, 48(8), 551-575.

Wang, X., \& Kerre, E. E. (2001). Reasonable properties for the ordering of fuzzy quantities (I). Fuzzy sets and systems, 118(3), 375-385.

Wei, S. H., \& Chen, S. M. (2009a). Fuzzy risk analysis based on interval-valued fuzzy numbers. Expert Systems with Applications, 36(2), 2285-2299.

Wei, S. H., \& Chen, S. M. (2009b). A new approach for fuzzy risk analysis based on similarity measures of generalized fuzzy numbers. Expert Systems with Applications, 36(1), 589-598.

Xu, Z., Shang, S., Qian, W., \& Shu, W. (2010). A method for fuzzy risk analysis based on the new similarity of trapezoidal fuzzy numbers. Expert Systems with Applications, 37(3), 1920-1927.

Zadeh, L. A. (1965). Fuzzy sets. Information and control, 8(3), 338-353.

Zhü, K. (2014). Fuzzy analytic hierarchy process: Fallacy of the popular methods. European Journal of Operational Research, 236(1), 209-217.

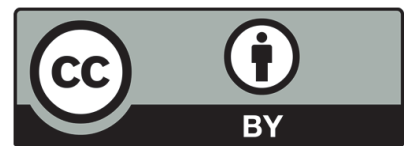

(C) 2019 by the authors; licensee Growing Science, Canada. This is an open access article distributed under the terms and conditions of the Creative Commons Attribution (CC-BY) license (http://creativecommons.org/licenses/by/4.0/). 